\title{
Cerebral Microbleeds and White Matter Hyperintensities in Cognitively Healthy Elderly: A Cross-Sectional Cohort Study Evaluating the Effect of Arterial Stiffness
}

\author{
Anna-Märta Gustavsson ${ }^{a, b}$ Erik Stomrud ${ }^{a}$, b Kasim Abul-Kasim ${ }^{c}$ \\ Lennart Minthon $^{\mathrm{a}, \mathrm{b}}$ Peter M. Nilsson ${ }^{\mathrm{d}}$ Oskar Hansson ${ }^{\mathrm{a}, \mathrm{b}}$ \\ Katarina Nägga ${ }^{a}$ b \\ ${ }^{\mathrm{a}}$ Memory Clinic, Skåne University Hospital, and ${ }^{\mathrm{b}} \mathrm{Clinical}$ Memory Research Unit and \\ ${ }^{c}$ Medical Radiology Unit, Department of Clinical Sciences Malmö, and d Department of \\ Clinical Sciences Malmö, Lund University, Malmö, Sweden
}

Key Words

Arterial stiffness · Cerebral microbleeds · Cognitive function · Healthy elderly · Pulse wave velocity $\cdot$ White matter hyperintensities

\section{Abstract}

Background: Arterial stiffness reflects the ageing processes in the vascular system, and studies have shown an association between reduced cognitive function and cerebral small vessel disease. Small vessel disease can be visualized as white matter hyperintensities (WMH) and lacunar infarcts but also as cerebral microbleeds on brain magnetic resonance imaging (MRI). We aimed to investigate if arterial stiffness influences the presence of microbleeds, WMH and cognitive function in a population of cognitively healthy elderly. Methods: The study population is part of the Swedish BioFinder study and consisted of 208 individuals without any symptoms of cognitive impairment, who scored >27 points on the Mini-Mental State Examination. The participants (mean age, 72 years; 59\% women) underwent MRI of the brain with visual rating of microbleeds and $\mathrm{WMH}$. Arterial stiffness was measured with carotid-femoral pulse wave velocity (cfPWV). Eight cognitive tests covering different cognitive domains were performed. Results: Microbleeds were detected in $12 \%$ and $\mathrm{WMH}$ in $31 \%$ of the participants. Mean ( \pm standard deviation, SD) cfPWV was $10.0( \pm 2.0) \mathrm{m} / \mathrm{s}$. There was no association between the presence of microbleeds and arterial stiffness. There was a positive association between arterial stiffness and WMH independent of age or sex (odds ratio, 1.58; 95\% confidence interval, 1.04-2.40, $p<0.05$ ), but the effect was attenuated when further adjustments for several cardiovascular risk factors were performed $(p>0.05)$. Cognitive performance was not associated with microbleeds, but individuals with WMH performed slightly worse than those with- 
out WMH on the Symbol Digit Modalities Test (mean \pm SD, $35 \pm 7.8$ vs. $39 \pm 8.1, p<0.05$ ). Linear regression revealed no direct associations between arterial stiffness and the results of the cognitive tests. Conclusions: Arterial stiffness was not associated with the presence of cerebral microbleeds or cognitive function in cognitively healthy elderly. However, arterial

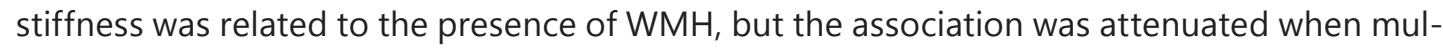
tiple adjustments were made. There was a weak negative association between $\mathrm{WMH}$ and performance in one specific test of attention. Longitudinal follow-up studies are needed to further assess the associations.

(C) 2015 S. Karger AG, Basel

\section{Introduction}

Cognitive impairment and dementia development is strongly associated with age, and the prevalence is increasing [1]. Cerebral small vessel disease, such as white matter hyperintensities (WMH) and microbleeds, is involved in the pathogenesis of cognitive impairment and dementia. Cardiovascular risk factors, and possibly arterial stiffness, also contribute to their development [2].

Arterial stiffening increases with age and reflects structural and cellular changes in the vascular system [3]. Measurement of carotid-femoral pulse wave velocity (cfPWV) is considered to be the gold standard method for estimating arterial stiffness [4]. Increased PWV has been associated with cardiovascular morbidity and mortality, independently of traditional cardiovascular risk factors [5]. In community-based samples, a negative association between increased cfPWV and cognitive performance in at least one domain has been found in some [6-8], but not in all studies $[9,10]$. Hence, these diverging results indicate a need for further clarification of the field. Numerous publications report a positive association between higher PWV and increased volume of WMH [6, 10-12]. Only 2 previous studies have investigated cfPWV and cerebral microbleeds. No associations were found in the general population, except in a subgroup of cases with uncontrolled hypertension [12]. The second study found no association in hypertensive patients [11].

Cerebral microbleeds are small perivascular haemorrhages and consist of iron deposits, mainly haemosiderin. They are seen as focal lesions on magnetic resonance imaging (MRI) and likely occur due to hypertensive arteriopathy or cerebral amyloid angiopathy that causes small vascular leaks $[13,14]$. Several studies report an association between the presence and number of microbleeds and impaired cognitive function. However, the cognitive domains affected differ between studies, but speed, attention and executive function seem to be predominantly affected [15-18].

WMH reflect damage to the deep subcortical and periventricular parts of the brain. Small vessel disease is believed to contribute to the development of ischaemia and tissue damage. WMH are considered to be associated with impairment in cognitive function. However, results from different studies are somewhat inconsistent, although most studies indicate a reduction in executive function and processing speed $[19,20]$.

Although chronological age is the strongest risk factor for impaired cognitive performance, many elderly people maintain a high level of intellectual ability throughout life. Education, physical activity and social interaction have been suggested to preserve cognitive function [2], theoretically compensating for different degrees of vascular risk factor load on the brain. The aim of the present study was to investigate the effects of $\mathrm{WMH}$ and cerebral microbleeds on cognitive results in an elderly group of individuals with well-preserved intellectual abilities. We also aimed to study the association between arterial stiffness and the presence of WMH and microbleeds in this particular group. 

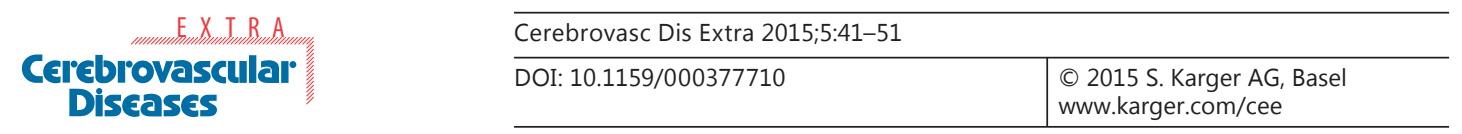

Gustavsson et al:: Cerebral Microbleeds and WMH in Cognitively Healthy Elderly:

A Cross-Sectional Cohort Study Evaluating the Effect of Arterial Stiffness

\section{Methods}

\section{Setting and Participants}

The cognitively healthy elderly cohort of the Swedish BioFinder study was recruited from Malmö Diet and Cancer Study (MDCS), a population-based cohort study conducted in Malmö, Sweden. The original cohort consisted of 28,449 individuals, and details of recruitment and data collection have been described previously [21]. Of these, 6,103 individuals were randomly selected to take part in the MDCS cardiovascular cohort, focusing on cardiovascular risk factors. The cardiovascular cohort was first examined between 1991 and 1996. Between 2007 and 2012, the MDCS cardiovascular cohort was re-examined with a participation rate of $76 \%$ of survivors $(n=3,734)$. Data on health status were collected by questionnaire, clinical examinations were performed and blood samples were drawn. Measurement of cfPWV and cognitive screening tests were added to the re-examination protocol. A total of 3,329 individuals underwent cognitive screening with the Mini-Mental State Examination (MMSE).

Subjects were randomly invited to participate in the Swedish BioFinder study as cognitively healthy elderly if they were aged $>60$ years, scored $>27$ points on MMSE and did not have any subjective cognitive impairment (www.biofinder.se). Exclusion criteria were history of transitory ischaemic attacks or stroke, presence of severe neurologic disease (e.g. Parkinson disease), severe psychiatric disease (e.g. severe depression or psychotic syndromes), dementia or mild cognitive impairment (MCI). Data were collected between 2009 and 2013 in accordance with a standard protocol. It was conducted by a physician and consisted of a review of medical history, extended cognitive testing and physical examination including neurologic and psychiatric evaluation. The baseline BioFinder protocol also included MRI of the brain and a lumbar puncture. The 208 participants who had undergone MRI by the time of the present study were included in the analyses. However, one person terminated MRI prematurely, and therefore the presence of cerebral microbleeds was estimated in 207 participants; WMH were graded in all 208 participants.

\section{Ethics}

All individuals gave written consent to participate in the study. Ethical approval for the study was given by the Ethical Committee of Lund University, Lund, Sweden.

\section{Arterial Stiffness}

cfPWV was estimated non-invasively with participants in the supine position after resting for $5 \mathrm{~min}$. The SphygmoCor system (AtCor Medical, Sydney, N.S.W., Australia) was used. cfPWV was computed in metres/second as the distance travelled by the wave, divided by the time it takes for the wave to travel the distance. The procedure has been described in more detail elsewhere [4]. In brief, the distance was estimated by body surface measurements. The carotid-femoral transit time was calculated using an electrocardiogram and a tonometer (pressure-sensitive probe) to register pulse curves. Data on cfPWV measurements were missing in cases of severe obesity and atrial fibrillation for technical reasons. Therefore, cfPWV data were available on 196 individuals.

\section{Magnetic Resonance Imaging}

MRI was performed on a Siemens 3T (TRIO) imaging system. Axial 4-mm-thick fluidattenuated inversion recovery and coronal 4-mm-thick gradient-echo $\mathrm{T} 2 *$-weighted images were evaluated in this study. The images were visually rated by a reader (A.-M.G.) trained by an experienced neuroradiologist (K.A.-K.). They were both blinded to all clinical data except age and sex. The reader evaluated the images independently, and in cases of uncertainty the final rating was done by a joined panel with the experienced neuroradiologist. 
Microbleeds were defined as round, hypointense lesions (signal voids) with a maximum diameter of $10 \mathrm{~mm}$ on gradient-echo T2*-weighted images. Flow voids due to blood vessels were disregarded. Symmetrical foci of low signal intensity in basal ganglia were considered to be calcifications in the globus pallidus.

The presence of WMH was evaluated on axial fluid-attenuated inversion recovery images and classified according to Fazeka's rating scale [22]. Periventricular hyperintensities (PVH) were graded as follows: 0 , absence; 1 , caps or pencil-thin lining; 2 , smooth halos, or 3, irregular PVH extending into the deep white matter. Deep WMH (DWMH) were graded as follows: 0, absence; 1 , punctuate foci; 2, beginning confluence of foci/coalescing, or 3, large confluent areas. PVH grade 0 or 1 and DWMH grade 0 or 1 were considered normal. The presence of either PVH or DWMH grade 2 or 3 was considered abnormal. Based on this, white matter changes were dichotomized into normal or abnormal, and abnormal was referred to as the presence of WMH.

\section{Covariates}

Blood pressure, anthropometric measurements and blood samples were collected during the MDCS re-examination. Blood pressure was measured twice after $5 \mathrm{~min}$ of rest in the supine position, immediately before the assessment of cfPWV. The equipment used was an OMRON M5-I IntelliSense (Omron Healthcare, Kyoto, Japan). Use of medication, prevalent diseases and lifestyle factors were derived from the MDCS questionnaire. Information on years of education, smoking status and prevalent diseases was collected during the BioFinder baseline visit. Cardiovascular disease was considered present when the participants reported myocardial infarction, angina pectoris or intermittent claudication. Ischaemic heart disease was considered present when the participants reported angina pectoris, myocardial infarction or coronary artery bypass operation.

\section{Cognitive Function}

Global cognitive function was assessed with the MMSE [23]. Episodic memory was tested with Alzheimer's Disease Assessment Scale delayed word recall [24]. The Symbol Digit Modalities Test (SDMT) [25] and Trail Making Test part A [26] were used to assess speed and attention. Executive function was tested with the Stroop test [27] and Trail Making Test part B [26]. A Quick Test of Cognitive Speed [28] was used to test executive function as well as attention and speed. Language fluency was evaluated with Letter S and Animal Fluency [29].

\section{Statistical Analysis}

All statistical analyses were performed using SPSS statistical software (version 22 for Windows). A p value $<0.05$ was considered significant. The independent samples t test was used to estimate group differences for numerical variables, and the $\chi^{2}$ test was used for categorical variables. If the expected count was $<5$ in one cell, the Fisher's exact test was used for categorical variables. The results are shown as mean \pm standard deviation (SD) or number (\%). The waist/hip ratio was logarithmically transformed due to the skewness of the data.

MRI variables were dichotomized into the presence or absence of WMH and microbleeds, respectively. Logistic regression models were used to assess the association between MRI data and arterial stiffness. cfPWV was transformed to a z score (individual cfPWV measure minus mean cfPWV measure divided by SD) when entered in the logistic regression models in order to evaluate the odds ratio (OR) per SD increase in cfPWV. The covariates were chosen based on previous literature on the assessment of cfPWV and risk factors for cerebral small vessel disease. Model 1 was adjusted for age and sex. Model 2 was further adjusted for mean 

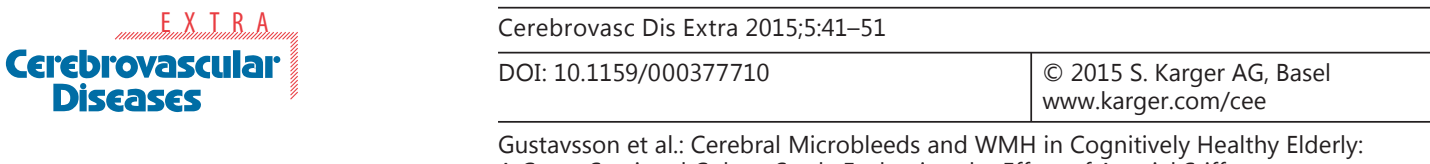

Gustavsson et al.: Cerebral Microbleeds and WMH in Cognitively Healthy Elderly:

A Cross-Sectional Cohort Study Evaluating the Effect of Arterial Stiffness

Table 1. Characteristics of the cognitively healthy elderly in the Swedish BioFinder study compared with the MDCS re-examination cohort

\begin{tabular}{llcrc}
\hline Characteristics & $\mathrm{n}$ & $\begin{array}{l}\text { Cognitively healthy } \\
(\mathrm{n}=208)\end{array}$ & $\begin{array}{l}\text { MDCS } \\
(\mathrm{n}=3,526)\end{array}$ & $\begin{array}{l}\mathrm{p} \\
\text { value }\end{array}$ \\
\hline Age, years & & $71 \pm 4.8$ & $73 \pm 5.6$ & $<0.001$ \\
Male sex & 3,734 & $85(41)$ & $1,437(41)$ & 0.98 \\
Higher education & 3,734 & $104(51)$ & $1,037(30)$ & $<0.001$ \\
Ever smoking & 3,619 & $127(62)$ & $1,842(54)$ & 0.033 \\
Waist/hip ratio & 3,614 & $0.89 \pm 0.1$ & $0.90 \pm 0.1$ & $0.36^{1}$ \\
Sedentary lifestyle & 3,696 & $5(2.4)$ & $298(8.6)$ & 0.002 \\
Systolic blood pressure, mm Hg & 3,679 & $137 \pm 17$ & $144 \pm 19$ & $<0.001$ \\
Diastolic blood pressure, mm Hg & 3,694 & $82 \pm 10$ & $83 \pm 10$ & 0.31 \\
Total cholesterol, mmol/l & 3,692 & $5.3 \pm 1.1$ & $5.2 \pm 1.1$ & 0.07 \\
Fasting plasma glucose, mmol/l & 3,692 & $5.9 \pm 1.0$ & $6.2 \pm 1.5$ & 0.006 \\
Cardiovascular disease & 3,694 & $12(6.7)$ & $11 . \pm 2.5$ & 0.001 \\
PWV, m/s & 3,127 & $10.0 \pm 2.0$ & $28 \pm 1.9$ & $<0.001$ \\
MMSE score & 3,056 & $29 \pm 0.8$ & $75 \pm 21$ & $<0.001$ \\
AQT, s & 3,329 & $65 \pm 11$ & $1,796(51)$ & 0.007 \\
Blood pressure-lowering medication & 3,310 & $86(41)$ & $1,072(30)$ & 0.36 \\
Lipid-lowering medication & 3,734 & $57(27)$ & $300(8.5)$ & 0.25 \\
Antidiabetic medication & 3,734 & $13(6.3)$ & $861(24)$ & $<0.001$ \\
Antiplatelet medication (ASA) & 3,734 & $28(14)$ & $165(4.7)$ & 0.82 \\
Anticoagulant medication (warfarin) & 3,734 & $9(4.3)$ & & 0.001 \\
\hline
\end{tabular}

Values are presented as mean \pm SD or $\mathrm{n}(\%)$. Group differences were analysed using the independent samples t test (numeric variables) or $\chi^{2}$ test (categorical variables). ASA = Acetylsalicylic acid; AQT = A Quick Test of Cognitive Speed.

${ }^{1} \mathrm{p}$ value on log data.

arterial pressure (MAP), heart rate, height, weight, smoking, total cholesterol, use of lipidlowering medication and use of blood pressure-lowering medication. MAP was calculated as diastolic pressure $+1 / 3$ (systolic pressure - diastolic pressure). The results are shown as the OR with 95\% confidence interval (CI).

Analysis of covariance was used to assess differences in cognitive performance between the groups with presence or absence of microbleeds and WMH, including age, sex and years of education as covariates. Linear regression models were used to explore any association between cognitive test results and cfPWV.

\section{Results}

\section{Participants}

The characteristics of the cognitively healthy elderly cohort are compared with the larger MDCS cohort in table 1. The differences in the two cohorts remained significant after adjustments for age. A history of smoking was less common among the cognitively healthy elderly, but the difference did not reach significance after adjustments for age (age adjusted $\mathrm{p}>0.05$ ).

The study population consisted of 208 individuals. The mean $( \pm$ SD) age at BioFinder study entry was $71.8( \pm 4.7)$ years and $59 \%$ were women. The mean $( \pm$ SD) cfPWV was 10.0 $( \pm 2.0) \mathrm{m} / \mathrm{s}$. Twenty-five of 207 individuals $(12 \%)$ had cerebral microbleeds and 65 of 208 

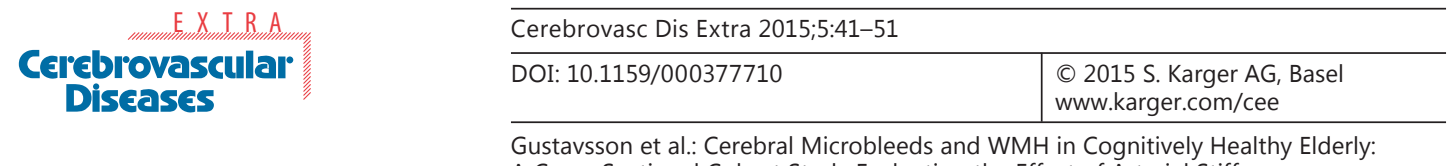

Gustavsson et al.: Cerebral Microbleeds and WMH in Cognitively Healthy Elderly:

A Cross-Sectional Cohort Study Evaluating the Effect of Arterial Stiffness

Table 2. Characteristics of the study population grouped by the presence of cerebral microbleeds (CMB) and WMH

\begin{tabular}{lcccccccc}
\hline Characteristics & $\mathrm{n}$ & $\begin{array}{l}\mathrm{CMB}- \\
(\mathrm{n}=182)\end{array}$ & $\begin{array}{l}\mathrm{CMB}+ \\
\mathrm{n}=25)\end{array}$ & $\begin{array}{l}\mathrm{p} \\
\text { value }\end{array}$ & $\mathrm{n}$ & $\begin{array}{l}\text { WMH- } \\
(\mathrm{n}=143)\end{array}$ & $\begin{array}{l}\text { WMH+ } \\
(\mathrm{n}=65)\end{array}$ & $\begin{array}{l}\mathrm{p} \\
\text { value }\end{array}$ \\
\hline Age, years & 207 & $72 \pm 4.6$ & $73 \pm 5.5$ & 0.36 & 208 & $71 \pm 4.6$ & $73 \pm 4.7$ & 0.001 \\
Male sex & 207 & $69(38)$ & $15(60)$ & 0.035 & 208 & $61(43)$ & $24(37)$ & 0.44 \\
Education, years & 207 & $12 \pm 3.6$ & $13 \pm 4.5$ & 0.29 & 208 & $12 \pm 3.7$ & $12 \pm 3.7$ & 0.65 \\
Currently smoking & 207 & $15(8.2)$ & $5(20)$ & 0.07 & 208 & $13(9.1)$ & $7(11)$ & 0.70 \\
Waist-to-hip ratio & 207 & $0.88 \pm 0.1$ & $0.93 \pm 0.1$ & 0.027 & 208 & $0.89 \pm 0.1$ & $0.89 \pm 0.1$ & 1.01 \\
Sedentary lifestyle & 206 & $4(2.2)$ & $1(4.0)$ & 0.48 & 207 & $3(2.1)$ & $2(3.1)$ & 0.65 \\
Systolic blood pressure, mm Hg & 207 & $137 \pm 17$ & $139 \pm 21$ & 0.62 & 208 & $135 \pm 17$ & $142 \pm 18$ & 0.005 \\
Diastolic blood pressure, mm Hg & 207 & $82 \pm 10$ & $85 \pm 12$ & 0.23 & 208 & $81 \pm 9.4$ & $84 \pm 12$ & 0.08 \\
Total cholesterol, mmol/l & 207 & $5.3 \pm 1.1$ & $5.3 \pm 1.0$ & 0.82 & 208 & $5.3 \pm 1.1$ & $5.4 \pm 1.1$ & 0.59 \\
Fasting plasma glucose, mmol/l & 207 & $5.9 \pm 1.0$ & $5.6 \pm 0.7$ & 0.10 & 208 & $5.9 \pm 1.1$ & $5.8 \pm 0.8$ & 0.33 \\
Ischemic heart disease & 207 & $11(6.0)$ & $4(16)$ & 0.09 & 208 & $10(7.0)$ & $5(7.7)$ & 1.00 \\
PWV, m/s & 195 & $10 \pm 2.0$ & $10 \pm 2.0$ & 0.76 & 196 & $9.7 \pm 1.9$ & $11 \pm 1.9$ & 0.002 \\
Blood pressure-lowering medication & 207 & $74(41)$ & $11(44)$ & 0.75 & 208 & $58(41)$ & $28(43)$ & 0.73 \\
Lipid-lowering medication & 207 & $49(27)$ & $8(32)$ & 0.59 & 208 & $41(29)$ & $16(25)$ & 0.54 \\
Antidiabetic medication & 207 & $12(6.6)$ & $1(4.0)$ & 1.00 & 208 & $10(7.0)$ & $3(4.6)$ & 0.76 \\
Antiplatelet medication (ASA) & 207 & $23(13)$ & $5(20)$ & 0.35 & 208 & $18(13)$ & $10(15)$ & 0.58 \\
Anticoagulant medication (warfarin) & 207 & $6(3.3)$ & $3(12)$ & 0.08 & 208 & $2(1.4)$ & $7(11)$ & 0.005 \\
\hline
\end{tabular}

Values are presented as mean \pm SD or $\mathrm{n}(\%)$. Group differences were analysed using the independent samples t test (numeric variables) or $\chi^{2}$ test/Fisher's exact test (categorical variables). ASA = Acetylsalicylic acid.

${ }^{1} \mathrm{p}$ value on $\log$ data.

Table 3. Association between arterial stiffness and the presence of cerebral microbleeds (CMB) and WMH

\begin{tabular}{|c|c|c|c|c|c|c|}
\hline \multirow[t]{2}{*}{ PWV per SD increase } & \multicolumn{3}{|l|}{$\mathrm{CMB}$} & \multicolumn{3}{|c|}{ WMH } \\
\hline & $\mathrm{n}$ & OR (95\% CI) & $\mathrm{p}$ value & $\mathrm{n}$ & OR (95\% CI) & $\mathrm{p}$ value \\
\hline Unadjusted & 195 & $1.09(0.63-1.88)$ & 0.75 & 196 & $1.83(1.24-2.72)$ & 0.003 \\
\hline Model 1 & 195 & $0.88(0.48-1.19)$ & 0.69 & 196 & $1.58(1.04-2.40)$ & 0.03 \\
\hline Model 2 & 195 & $0.67(0.31-1.44)$ & 0.31 & 196 & $1.29(0.81-2.08)$ & 0.29 \\
\hline
\end{tabular}

Logistic regression models with MRI-derived markers as dependent variables. Model 1 adjusted for age and sex. Model 2 adjusted for age, sex, MAP, heart rate, height, weight, smoking, total cholesterol, use of lipidlowering medication and use of blood pressure-lowering medication.

(31\%) had WMH on MRI. Six individuals (3\%) had multiple microbleeds and 10 (5\%) had both WMH and microbleeds.

Univariate statistical analysis of the study population, grouped by the presence of microbleeds and $\mathrm{WMH}$, is presented in table 2 . Individuals with microbleeds were more often male $(p<0.05)$ and had a higher waist/hip ratio $(p<0.05)$. No other differences could be detected between individuals with or without microbleeds (table 2). Individuals with WMH were significantly older $(p=0.001)$, had higher systolic blood pressure $(p=0.005)$, higher PWV $(p=0.002)$ and were more frequently prescribed warfarin $(p=0.005)$. No other differences between individuals with or without WMH were found (table 2). 

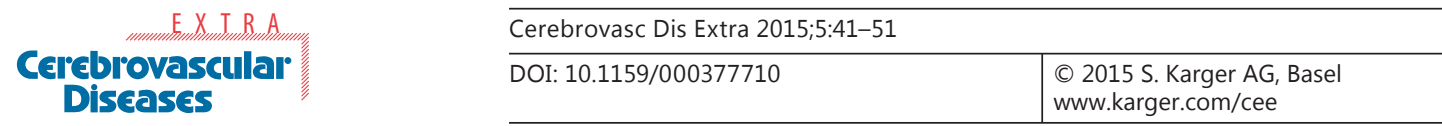

Gustavsson et al:: Cerebral Microbleeds and WMH in Cognitively Healthy Elderly

A Cross-Sectional Cohort Study Evaluating the Effect of Arterial Stiffness

Table 4. Cognitive test results grouped by the presence of cerebral microbleeds (CMB) and WMH

\begin{tabular}{lcccccccc}
\hline Cognitive test results & $\mathrm{n}$ & $\begin{array}{l}\mathrm{CMB}- \\
(\mathrm{n}=182)\end{array}$ & $\begin{array}{l}\mathrm{CMB}+ \\
(\mathrm{n}=25)\end{array}$ & $\begin{array}{l}\mathrm{p} \\
\text { value }\end{array}$ & $\mathrm{n}$ & $\begin{array}{l}\text { WMH- } \\
(\mathrm{n}=143)\end{array}$ & $\begin{array}{l}\text { WMH+ } \\
(\mathrm{n}=65)\end{array}$ & $\begin{array}{l}\mathrm{p} \\
\text { value }\end{array}$ \\
\hline MMSE, n & 207 & $29 \pm 0.9$ & $29 \pm 0.8$ & 0.66 & 208 & $29 \pm 0.9$ & $29 \pm 0.9$ & 0.36 \\
AQT, s & 207 & $63 \pm 11$ & $62 \pm 8.8$ & 0.54 & 208 & $63 \pm 12$ & $64 \pm 8.4$ & 0.83 \\
ADAS delayed word recall, $\mathrm{n}$ & 207 & $8.2 \pm 1.8$ & $7.9 \pm 1.9$ & 0.72 & 208 & $8.1 \pm 1.9$ & $8.2 \pm 1.6$ & 0.51 \\
SDMT, n & 207 & $38 \pm 8.2$ & $36 \pm 8.5$ & 0.42 & 208 & $39 \pm 8.1$ & $35 \pm 7.8$ & 0.049 \\
Stroop, s & 207 & $28 \pm 5.9$ & $27 \pm 6.0$ & 0.34 & 208 & $28 \pm 6.2$ & $28 \pm 5.4$ & 0.22 \\
TMT-A, s & 207 & $44 \pm 14$ & $42 \pm 11$ & 0.33 & 208 & $42 \pm 23$ & $47 \pm 15$ & 0.23 \\
TMT-B, s & 164 & $96 \pm 50$ & $92 \pm 35$ & 0.64 & 165 & $97 \pm 54$ & $93 \pm 35$ & 0.15 \\
Letter S, n & 207 & $16 \pm 5.6$ & $15 \pm 5.8$ & 0.53 & 208 & $16 \pm 5.4$ & $16 \pm 6.2$ & 0.97 \\
Animal fluency, n & 207 & $22 \pm 5.1$ & $22 \pm 6.5$ & 0.54 & 208 & $22 \pm 5.1$ & $21 \pm 5.7$ & 0.98 \\
\hline
\end{tabular}

Values are presented as mean \pm SD. Group differences were analysed using analysis of covariance, adjusted for age, sex and years of education. AQT = A Quick Test of Cognitive Speed; ADAS = Alzheimer's Disease Assessment Scale; TMT-A/B = Trail Making Test part A/B; $\mathrm{n}=$ number of correct answers.

\section{Association between cfPWV and Microbleeds or WMH}

PWV measures were available for 195 individuals with microbleed ratings and 196 with WMH ratings. There was no association between the presence of microbleeds and arterial stiffness in the univariate or adjusted logistic regression models (table 3). There was an association between arterial stiffness and WMH in the crude analysis (unadjusted OR, 1.83; 95\% CI, 1.24-2.72; $\mathrm{p}=0.003$ ). The association was attenuated, but remained significant, after adjustment for age and sex (OR, 1.58; 95\% CI, 1.04-2.40; $\mathrm{p}=0.03$ ). When further adjustments were made, the association was no longer statistically significant (table 3 ).

\section{Cognitive Test Results}

There was no difference in cognitive performance between individuals with or without microbleeds (table 4). Individuals with WMH performed slightly worse than individuals without WMH on the SDMT (mean \pm SD; $35 \pm 7.8$ correct answers vs. $39 \pm 8.1$ correct answers; $\mathrm{p}$ value $=0.049$ ). No other differences between those with or without WMH were found (table 4). All models were adjusted for age, sex and years of education.

Using linear regression models, we found no statistically significant associations between arterial stiffness and different cognitive test results in the study population. All models were adjusted for age, sex and years of education (data not shown).

\section{Discussion}

In this observational study of cognitively healthy individuals, arterial stiffness was not related to the presence of cerebral microbleeds, and their presence did not affect cognitive performance. Higher measures of arterial stiffness were related to the presence of WMH, and individuals with WMH had a reduced outcome in one test reflecting the cognitive domains of speed and attention (SDMT).

Our neutral results regarding microbleeds are in line with previous studies by Henskens et al. [11] and Poels et al. [12] and therefore support these findings also in cognitively healthy individuals. Studies that have shown a positive association between arterial stiffness and microbleeds used brachial-ankle PWV [30,31], which is not considered to be as reliable as 
cfPWV measurements because the first method includes not only elastic arteries but also muscular arteries in the limbs [4].

On assessment, microbleeds are often categorized according to location, separating strictly lobar microbleeds from deep or infratentorial microbleeds. Poels et al. [12] found an association between arterial stiffness and deep or infratentorial microbleeds in individuals with uncontrolled hypertension. This may be explained by the theoretical assumption that deep or infratentorial microbleeds appear due to hypertensive vasculopathy, whereas lobar microbleeds are a result of amyloid angiopathy [32]. Other studies also investigated the number of microbleeds when exploring associations with cognitive performance, and multiple microbleeds were associated with worse cognitive performance than solitary microbleeds $[15,16]$. In our data, we did not apply these categorizations due to the relatively low number of cases with microbleeds $(n=25)$ and the low prevalence of multiple microbleeds $(n=6)$. The lack of significant associations between microbleeds and arterial stiffness and cognition is therefore mainly based on the presence of solitary microbleeds.

Our results on a positive association between increasing cfPWV and the presence of WMH remained significant when age and sex were included in the logistic regression model. However, the effects did not remain significant when MAP, heart rate and other cardiovascular covariates were entered into the model. Other studies have shown more robust associations $[6,11,12,33]$. These discrepancies may be due to their larger study populations, evaluation of high-risk populations and assessment of WMH volume instead of visual rating.

Arterial stiffness was not associated with cognitive performance in our population. A recent review concluded that the current evidence is highly suggestive of an association between arterial stiffness and cognitive function but it is not conclusive due to the imprecise measurements often used, such as brachial-ankle PWV (as discussed above) and MMSE [34]. MMSE is mainly a screening test and is not precise enough to test more detailed cognitive function. The domains affected also differ between studies $[6,7,34]$. This may indicate that the associations are weak as a result of the complexity of brain ageing, cognitive reserve and its effect on cognitive performance. Because our population was selected based on the absence of cognitive disabilities, it is probable that any cross-sectional association with cognitive performance is difficult to establish, even though the inclusion criteria of MMSE score $>27$ does not rule out impairments in more domain-specific tests.

The cognitively healthy elderly cohort of the Swedish BioFinder study consists of a selected population of elderly individuals with a higher level of education, less cardiovascular disease, and who are more physically active than the general population. This might be regarded as a selection bias, but is probably an underlying prerequisite for preserving cognitive function. Since the aim of the study was to investigate a group of cognitively healthy elderly, these differences from the general population are anticipated and support the notion that this is the intended study population.

Although cognitive impairment is common among the elderly, a majority have generally preserved cognitive abilities. Many studies are carried out in memory clinic settings, thus focusing on diseased brains. Our study aimed to focus on individuals who did not have subjective or objective cognitive impairment in order to study whether previously reported disease-generating mechanisms are clinically important in this population. Our findings imply that elderly people with structural brain changes, evident on MRI, may perform well on cognitive tests and lead a symptom-free life. This supports the assumption that cognitive reserve plays an important role in cognitive function and further underlines the importance of combining neuroimaging with symptom analyses and detailed cognitive testing in clinical practice when the aim is to differentiate between normal cognitive aging and MCI. It is of great significance to further evaluate these individuals longitudinally in order to pursue cognitive 
assessment over time and evaluate if markers of cerebral small vessel disease on MRI predict conversion to $\mathrm{MCI}$ and dementia.

One limitation of the study is its cross-sectional design, which does not address causality. In addition, the grading of structural brain damage was done visually, and this might introduce subjectivity in the grading. Susceptibility-weighted imaging has been suggested to be more sensitive for the detection of microbleeds compared to T2*-weighted MRI used in our study. This may possibly underestimate the prevalence of microbleeds. The study sample is relatively small, and this prevented us from doing separate analyses based on the number and location of microbleeds, as previously mentioned. Furthermore, including multiple covariates in the analyses with few positive events might attenuate the statistical effects and hence possibly underestimate the strength of the underlying association.

\section{Conclusions}

In this cross-sectional study of a population of cognitively healthy individuals, arterial stiffness did not influence the presence of cerebral microbleeds or cognitive function. Arterial stiffness was related to the presence of $\mathrm{WMH}$, but the association was attenuated when multiple adjustments were made. There was a weak negative association between WMH and performance in one specific test of attention. Longitudinal follow-up studies are ongoing to further assess the role of microbleeds and WMH on the development of cognitive decline.

\section{Acknowledgements}

The authors thank research nurses Malin Otthén and Karina Simonsen in the BioFinder study. We also thank Margaretha Persson and Gerd Östling for the study logistics and examinations in the MDCS re-examination study, and Erik D. Nilsson and Carina Wattmo for their valuable input during the writing process. The study was supported by the European Research Council, the Swedish Research Council, the Crafoord Foundation, the Swedish Brain Foundation, The Swedish Alzheimer foundation, the Skåne University Hospital Foundation and the regional agreement on medical training and clinical research (ALF) between Skåne County Council and Lund University. The funding sources had no role in the design and conduct of the study, in the collection, analysis, interpretation of the data, or in the preparation, review or approval of the manuscript.

\section{Disclosure Statement}

The authors have no conflicts of interest to declare.

\section{References}

1 Ferri CP, Prince M, Brayne C, Brodaty H, Fratiglioni L, Ganguli M, Hall K, Hasegawa K, Hendrie H, Huang Y, Jorm A, Mathers C, Menezes PR, Rimmer E, Scazufca M: Global prevalence of dementia: a Delphi consensus study. Lancet 2005;366:2112-2117.

-2 Gorelick PB, Scuteri A, Black SE, Decarli C, Greenberg SM, Iadecola C, Launer LJ, Laurent S, Lopez OL, Nyenhuis D, Petersen RC, Schneider JA, Tzourio C, Arnett DK, Bennett DA, Chui HC, Higashida RT, Lindquist R, Nilsson PM, Roman GC, Sellke FW, Seshadri S: Vascular contributions to cognitive impairment and dementia: a statement for healthcare professionals from the American Heart Association/American Stroke Association. Stroke 2011;42:2672-2713. 


\begin{tabular}{l|l}
\hline \multicolumn{2}{l|}{ Cerebrovasc Dis Extra 2015;5:41-51 } \\
\hline DOI: $10.1159 / 000377710$ & $\begin{array}{l}\text { C 2015 S. Karger AG, Basel } \\
\text { www.karger.com/cee }\end{array}$ \\
\hline
\end{tabular}

Gustavsson et al.: Cerebral Microbleeds and WMH in Cognitively Healthy Elderly: A Cross-Sectional Cohort Study Evaluating the Effect of Arterial Stiffness

3 Laurent S, Boutouyrie P, Lacolley P: Structural and genetic bases of arterial stiffness. Hypertension 2005;45: 1050-1055.

4 Laurent S, Cockcroft J, Van Bortel L, Boutouyrie P, Giannattasio C, Hayoz D, Pannier B, Vlachopoulos C, Wilkinson I, Struijker-Boudier H: Expert consensus document on arterial stiffness: methodological issues and clinical applications. Eur Heart J 2006;27:2588-2605.

5 Ben-Shlomo Y, Spears M, Boustred C, May M, et al: Aortic pulse wave velocity improves cardiovascular event prediction: an individual participant meta-analysis of prospective observational data from 17,635 subjects. J Am Coll Cardiol 2014;63:636-646.

6 Mitchell GF, van Buchem MA, Sigurdsson S, Gotal JD, Jonsdottir MK, Kjartansson O, Garcia M, Aspelund T, Harris TB, Gudnason V, Launer LJ: Arterial stiffness, pressure and flow pulsatility and brain structure and function: the Age, Gene/Environment Susceptibility - Reykjavik Study. Brain 2011;134:3398-3407.

7 Poels MM, van Oijen M, Mattace-Raso FU, Hofman A, Koudstaal PJ, Witteman JC, Breteler MM: Arterial stiffness, cognitive decline, and risk of dementia: the Rotterdam Study. Stroke 2007;38:888-892.

-8 Nilsson ED, Elmstahl S, Minthon L, Nilsson PM, Pihlsgard M, Tufvesson E, Nagga K: Nonlinear association between pulse wave velocity and cognitive function: a population-based study. J Hypertens 2014;32:21522157.

-9 Singer J, Trollor JN, Crawford J, O’Rourke MF, Baune BT, Brodaty H, Samaras K, Kochan NA, Campbell L, Sachdev PS, Smith E: The association between pulse wave velocity and cognitive function: The Sydney Memory and Ageing Study. PLoS One 2013;8:e61855.

-10 Tsao CW, Seshadri S, Beiser AS, Westwood AJ, Decarli C, Au R, Himali JJ, Hamburg NM, Vita JA, Levy D, Larson MG, Benjamin EJ, Wolf PA, Vasan RS, Mitchell GF: Relations of arterial stiffness and endothelial function to brain aging in the community. Neurology 2013;81:984-991.

-11 Henskens LH, Kroon AA, van Oostenbrugge RJ, Gronenschild EH, Fuss-Lejeune MM, Hofman PA, Lodder J, de Leeuw PW: Increased aortic pulse wave velocity is associated with silent cerebral small-vessel disease in hypertensive patients. Hypertension 2008;52:1120-1126.

12 Poels MM, Zaccai K, Verwoert GC, Vernooij MW, Hofman A, van der Lugt A, Witteman JC, Breteler MM, MattaceRaso FU, Ikram MA: Arterial stiffness and cerebral small vessel disease: The Rotterdam Scan Study. Stroke 2012;43:2637-2642.

13 Greenberg SM, Vernooij MW, Cordonnier C, Viswanathan A, Al-Shahi Salman R, Warach S, Launer LJ, van Buchem MA, Breteler MM: Cerebral microbleeds: a guide to detection and interpretation. Lancet Neurol 2009; 8:165-174.

14 Charidimou A, Krishnan A, Werring DJ, Rolf Jager H: Cerebral microbleeds: a guide to detection and clinical relevance in different disease settings. Neuroradiology 2013;55:655-674.

15 Poels MM, Ikram MA, van der Lugt A, Hofman A, Niessen WJ, Krestin GP, Breteler MM, Vernooij MW: Cerebral microbleeds are associated with worse cognitive function: The Rotterdam Scan Study. Neurology 2012;78: 326-333.

16 Qiu C, Cotch MF, Sigurdsson S, Jonsson PV, Jonsdottir MK, Sveinbjrnsdottir S, Eiriksdottir G, Klein R, Harris TB, van Buchem MA, Gudnason V, Launer LJ: Cerebral microbleeds, retinopathy, and dementia: the Ages-Reykjavik Study. Neurology 2010;75:2221-2228.

-17 van Norden AG, van den Berg HA, de Laat KF, Gons RA, van Dijk EJ, de Leeuw FE: Frontal and temporal microbleeds are related to cognitive function: the Radboud University Nijmegen diffusion Tensor and Magnetic Resonance Cohort (RUN DMC) study. Stroke 2011;42:3382-3386.

18 Yakushiji Y, Nishiyama M, Yakushiji S, Hirotsu T, Uchino A, Nakajima J, Eriguchi M, Nanri Y, Hara M, Horikawa E, Kuroda Y: Brain microbleeds and global cognitive function in adults without neurological disorder. Stroke 2008;39:3323-3328.

19 Debette S, Markus HS: The clinical importance of white matter hyperintensities on brain magnetic resonance imaging: systematic review and meta-analysis. BMJ 2010;341:c3666.

20 Poggesi A, Pantoni L, Inzitari D, Fazekas F, Ferro J, O’Brien J, Hennerici M, Scheltens P, Erkinjuntti T, Visser M, Langhorne P, Chabriat H, Waldemar G, Wallin A, Wahlund A: 2001-2011: a decade of the LADIS (leukoaraiosis and disability) study: what have we learned about white matter changes and small-vessel disease? Cerebrovasc Dis 2011;32:577-588.

-21 Manjer J, Carlsson S, Elmstahl S, Gullberg B, Janzon L, Lindstrom M, Mattisson I, Berglund G: The Malmo Diet and Cancer Study: representativity, cancer incidence and mortality in participants and non-participants. Eur J Cancer Prev 2001;10:489-499.

-22 Fazekas F, Chawluk JB, Alavi A, Hurtig HI, Zimmerman RA: MR signal abnormalities at 1.5 T in Alzheimer's dementia and normal aging. AJR Am J Roentgenol 1987;149:351-356.

-23 Folstein MF, Folstein SE, McHugh PR: 'Mini-mental state'. A practical method for grading the cognitive state of patients for the clinician. J Psychiatr Res 1975;12:189-198.

-24 Rosen WG, Mohs RC, Davis KL: A new rating scale for Alzheimer's disease. Am J Psychiatry 1984;141:13561364.

25 Wechsler D: WAIS-R Manual. New York, Psychological Corporation, 1981.

26 Reitan R: The Halstead-Reitan Neuropsychological Test Battery. Tucson, Neuropsychology Press, 1985.

27 Spreen O, Strauss E: A Compendium of Neuropsychological Tests. New York, Oxford University Press, 1998.

28 Jacobson JM, Nielsen NP, Minthon L, Warkentin S, Wiig EH: Multiple rapid automatic naming measures of cognition: normal performance and effects of aging. Percept Motor Skills 2004;98:739-753. 
29 Crossley M, D’Arcy C, Rawson NS: Letter and category fluency in community-dwelling Canadian seniors: a comparison of normal participants to those with dementia of the Alzheimer or vascular type. J Clin Exp Neuropsychol 1997;19:52-62.

-30 Song TJ, Kim J, Kim YD, Nam HS, Lee HS, Nam CM, Heo JH: The distribution of cerebral microbleeds determines their association with arterial stiffness in non-cardioembolic acute stroke patients. Eur J Neurol 2014;21: 463-469.

-31 Ochi N, Tabara Y, Igase M, Nagai T, Kido T, Miki T, Kohara K: Silent cerebral microbleeds associated with arterial stiffness in an apparently healthy subject. Hypertens Res 2009;32:255-260.

-32 Poels MM, Vernooij MW, Ikram MA, Hofman A, Krestin GP, van der Lugt A, Breteler MM: Prevalence and risk factors of cerebral microbleeds: an update of the Rotterdam Scan Study. Stroke 2010;41:S103-106.

33 Coutinho T, Turner ST, Kullo IJ: Aortic pulse wave velocity is associated with measures of subclinical target organ damage. JACC Cardiovasc Imaging 2011;4:754-761.

-34 Singer J, Trollor JN, Baune BT, Sachdev PS, Smith E: Arterial stiffness, the brain and cognition: a systematic review. Ageing Res Rev 2014;15C:16-27. 